Research Paper

\title{
A NOTE ON LOCAL ENTROPY OF RANDOM DYNAMICAL SYSTEMS
}

\author{
MEHDI RAHIMI*, AHMAD SHAKOURI, AND MOHAMMAD MOHAMMADI ANJEDANI
}

\begin{abstract}
In this paper, we review some properties of the entropy of random dynamical systems. We define a local entropy map for random dynamical systems and study some of its properties. We extract the entropy of random dynamical systems from the introduced map.
\end{abstract}

MSC(2010): 37A35; 37H99.

Keywords: Entropy, local entropy, random dynamical systems.

\section{INTRODUCTION}

Random dynamical systems, abbreviated by RDS, are generalizations of deterministic dynamical systems, in the sense that, at each time step a transformation is chosen randomly from a given family according to some probability distribution [1]. Indeed, an RDS is characterized by a state space $X$, a probability space $(\Omega, \mathcal{F}, \mathbb{P})$, with a usually $\mathbb{P}$-ergodic map $\theta: \Omega \rightarrow \Omega$, for which the assignment $\omega \longmapsto \varphi_{\omega}$ is done randomly.

Many subjects in the ergodic theory of deterministic dynamical systems are discussed for RDSs. Invariant measures, ergodic measures, random ergodic theorems and entropy of random dynamical systems are among the concepts which are discussed for RDSs [2, 31, 11]. These concepts are mainly motivated from deterministic dynamical systems.

Local approaches to the entropy of dynamical systems are discussed and studied extensively $[30,18,5,6,29,20,16]$. Motivated by localization of entropy in deterministic dynamical systems [25, 26, 27], we present a local view to the entropy of RDSs. To do this, we introduce a map which is defined at each point of the space $\Omega \times X$ and is nearly related to the entropy of the RDS defined on $\Omega \times X$, in the sense that the entropy of an RDS may be extracted by integration of the introduced map.

In Section 2, we provide some preliminary concepts and backgrounds which are necessary for the rest of the paper. In Section 3, we present a local approach to the entropy of RDSs. We conclude the paper with a concluding remark.

\section{Preliminary facts}

In this section, we review some preliminary concepts and facts. The definitions and concepts discussed in this section are mainly from [7, 8]. One may see $[1,11,12,14]$ for general theory of RDS. Let $X$ be a compact metric space with the Borel $\sigma$-algebra $\mathcal{B}_{X}$. We also write

Date: Received: December 22, 2020 , Accepted:May 5, 2021.

${ }^{*}$ Corresponding author. 
$C(X, X)$ for the set of continuous functions $f: X \rightarrow X$ with $C^{0}$-topology. Let also $(\Omega, \mathcal{F}, \mathbb{P})$ be a countably generated probability space, and $\theta: \Omega \rightarrow \Omega$ an invertible $\mathbb{P}$-ergodic system.

A measurable map $\varphi:(\Omega, \mathcal{F}, \mathbb{P}) \rightarrow C(X, X)$ defined by $\omega \mapsto \varphi_{\omega}$ is called a random dynamical system on $X$ over $(\Omega, \mathcal{F}, \mathbb{P}, \theta)$.

The skew product transformation associated with a random dynamical system $\varphi$ is defined by

$$
\begin{gathered}
\Phi: \Omega \times X \rightarrow \Omega \times X \\
(\omega, x) \mapsto\left(\theta(\omega), \varphi_{\omega}(x)\right) .
\end{gathered}
$$

For $n \geq 1$ we also write

$$
\varphi_{\omega}^{n}:=\left\{\begin{array}{cc}
i d_{X} & n=0 \\
\varphi_{\theta^{n-1}(\omega)} \circ \ldots \circ \varphi_{\theta(\omega)} \circ \varphi_{\omega} & n \geq 1
\end{array}\right.
$$

If $\pi_{\Omega}: \Omega \times X \rightarrow \Omega$ is the projection on $\Omega$, we easily have $\pi_{\Omega} \circ \Phi=\theta \circ \pi_{\Omega}$.

A probability measure $\mu$ on $\left(\Omega \times X, \mathcal{F} \times \mathcal{B}_{X}\right)$ is said to be $\varphi$-invariant if

(1) $\mu$ is invariant under $\Phi$.

(2) $\pi_{\Omega}^{*} \mu=\mathbb{P}$, where $\pi_{\Omega}^{*} \mu: \mathcal{F} \rightarrow[0,1]$ defined by $\pi_{\Omega}^{*} \mu(\Lambda)=\mu\left(\pi_{\Omega}^{-1}(\Lambda)\right)$ is called the marginal of $\mu$.

We denote the collection of $\varphi$-invariant measures by $I_{\mathbb{P}}(\varphi)$.

An invariant measure $\mu$ on $\mathcal{F} \times \mathcal{B}_{X}$ is called $\varphi$-ergodic if $\mu$ is $\Phi$-ergodic. The collection of all $\varphi$-ergodic measures is denoted by $E_{\mathbb{P}}(\varphi)$.

2.1. Disintegration of a measure. In the rest of the paper, we assume that $\mu$ disintegrates with respect to $\mathbb{P}$, i.e, there is a family of conditional probability measures $\left\{\mu_{\omega}\right\}_{\omega \in \Omega}$ on $\mathcal{B}_{X}$ such that $d \mu(\omega, x)=d \mu_{\omega}(x) d \mathbb{P}(\omega)$. Indeed, If $X$ is a Polish space, then any $\varphi$-invariant measure $\mu$ has a unique disintegration [1].

Note that, the previous condition is equivalent to

$$
\mu(D)=\int_{\Omega} \int_{X} \chi_{D}(\omega, x) d \mu(x) d \mathbb{P}(\omega)
$$

for $D \in \mathcal{F} \times \mathcal{B}_{X}$.

Also, the condition $d \mu(\omega, x)=d \mu_{\omega}(x) d \mathbb{P}(\omega)$ results in the following:

(1) $\int_{\Omega \times X} f d \mu=\int_{\Omega} \int_{X} f(\omega, x) d \mu_{\omega}(x) d \mathbb{P}(\omega)$ for all $f \in L^{1}(\mu)$.

(2) If $A \in \mathcal{F} \times \mathcal{B}_{X}$ and $\omega \in \Omega$, then

$$
\mu(A)=\int_{\Omega} \mu_{\omega}\left(A_{\omega}\right) d \mathbb{P}(\omega)
$$

where $A_{\omega}=\{x \in X:(\omega, x) \in A\}$ is the $\omega$-section of $A$.

2.2. Weak* topology. Denote $\|f\|_{b}:=\sup _{x \in X}|f(x)|$ for any $f \in C(X)$. A function $f: \Omega \rightarrow$ $C(X), \omega \mapsto f_{\omega}=f(\omega,$.$) is called measurable if the function (\omega, x) \mapsto f_{\omega}(x)=f(\omega, x)$ is measurable. The set of all measurable functions $f: \Omega \rightarrow C(X)$ with $\|f\|:=\int_{\Omega}\left\|f_{\omega}\right\|_{b} d \mathbb{P}(\omega)<$ $+\infty$ is denoted by $L_{\mathbb{P}}^{1}(\Omega, C(X))$. It is easily seen that $L_{\mathbb{P}}^{1}(\Omega, C(X))$ is a Banach space.

Let $\mathcal{M}(X)$ be the set of all complex Borel measures on $X$, equipped by the norm $\|\cdot\|=$ $|\cdot|(X)$. Now let $L_{\mathbb{P}}^{\infty}(\Omega, \mathcal{M}(X))$ be the set of functions $\mu: \Omega \rightarrow \mathcal{M}(X), \omega \mapsto \mu_{\omega}$ with $\|\mu\|_{\infty}<+\infty$ where

$$
\|\mu\|_{\infty}:=\inf \left\{M>0:\left\|\mu_{\omega}\right\|=\left|\mu_{\omega}\right|(X)<M, \text { for } \mathbb{P} \text {.a.e. } \omega \text { in } \Omega\right\}
$$


Since $\mathcal{M}(X)$ is the dual of $C(X)$ we will have:

$$
L_{\mathbb{P}}^{1}(\Omega, C(X))^{*}=L_{\mathbb{P}}^{\infty}(\Omega, \mathcal{M}(X)) .
$$

This induces the weak ${ }^{*}$ topology on $I_{\mathbb{P}}(\mu) \subset L_{\mathbb{P}}^{\infty}(\Omega, \mathcal{M}(X))$. Indeed, for $\left\{\mu_{n}\right\}_{n \geq 1}$ and $\mu$ in $I_{\mathbb{P}}(\mu)$ we have:

$$
\mu_{n} \rightarrow \mu \Longleftrightarrow \forall f \in L_{\mathbb{P}}^{1}(\Omega, C(X)) \quad \int_{\Omega \times X} f d \mu_{n} \rightarrow \int_{\Omega \times X} f d \mu .
$$

In light of Krein-Milman theorem, we have the following proposition.

Proposition 2.1. Let $X$ be a compact metric space and $\varphi$ be a continuous random dynamical system over $(\Omega, \mathcal{F}, \mathbb{P}, \theta)$. Then

(1) $I_{\mathbb{P}}(\varphi)$ is a non-empty convex compact subset of $L_{\mathbb{P}}^{\infty}(\Omega, \mathcal{M}(X))$.

(2) The set of extreme points of $I_{\mathbb{P}}(\varphi)$ is equal to $E_{\mathbb{P}}(\varphi)$.

For a compact metric space $X, C(X)$ is separable. Since $(\Omega, \mathcal{F}, \mathbb{P})$ is countably generated, $L_{\mathbb{P}}^{1}(\Omega, C(X))$ is also separable, so the topology of weak convergence of the compact Hausdorff space $L_{\mathbb{P}}^{\infty}(\Omega, \mathcal{M}(X))$ will be metrizable. Therefore, applying the Choquet's theorem [19] to $I_{\mathbb{P}}(\varphi)$, we have the following ergodic decomposition for the elements of $I_{\mathbb{P}}(\varphi)$.

Proposition 2.2. Suppose that $\mathbb{P}$ is $\theta$-ergodic. Then for each $\mu \in I_{\mathbb{P}}(\varphi)$ there exists a unique Borel probability measure $\tau=\tau_{\mu}$ on the $\sigma$-algebra of Borel subsets of $I_{\mathbb{P}}(\varphi)$ with $\tau_{\mu}\left(E_{\mathbb{P}}(\varphi)\right)=1$ and that

$$
\int_{\Omega \times X} f d \mu=\int_{E_{\mathbb{P}}(\varphi)}\left(\int_{\Omega \times X} f d \nu\right) d \tau_{\mu}(\psi)
$$

for any $f \in L_{\mathbb{P}}^{1}(\Omega, C(X))$.

Under the assumptions of the previous proposition, we write $\mu=\int_{E_{\mathbb{P}}(\varphi)} \nu d \tau_{\mu}(\nu)$ and call it the ergodic decomposition of $\mu$.

2.3. The entropy of random dynamics. The entropy of dynamical systems was first defined in [13, 32], and then was studied from other view points in [10, 17, 20, 28, 29].

Using the ideas in classical dynamical systems, this quantity is formulated for random dynamical systems [7, 8, 35, 34].

Let $\xi$ be a finite measurable partition of $\Omega \times X$. For $\omega \in \Omega$, set $\xi_{\omega}=\left\{D_{\omega}\right\}_{\omega \in \Omega}$, where $D_{\omega}$ is the $\omega$-section of $D$. Clearly, $\xi_{\omega}$ is a finite partition of $X$.

For $\omega \in \Omega$, set

$$
H_{\mu_{\omega}}\left(\bigvee_{i=0}^{n-1}\left(\varphi_{\omega}^{i}\right)^{-1} \xi_{\theta^{i}(\omega)}\right):=-\sum_{A \in \bigvee_{i=0}^{n-1}\left(\varphi_{\omega}^{i}\right)^{-1} \xi_{\theta^{i}(\omega)}} \mu_{\omega}(A) \log \mu_{\omega}(A)
$$

and

$$
h_{\mu}^{r}(\varphi, \xi):=\lim _{n \rightarrow \infty} \frac{1}{n} \int_{\Omega} H_{\mu_{\omega}}\left(\bigvee_{i=0}^{n-1}\left(\varphi_{\omega}^{i}\right)^{-1} \xi_{\theta^{i}(\omega)}\right) d \mathbb{P}(\omega) .
$$

Note that, the limit in (2.1) exists. Also, if $\mathbb{P}$ is $\theta$-ergodic, then

$$
h_{\mu}^{r}(\varphi, \xi)=\lim _{n \rightarrow \infty} \frac{1}{n} H_{\mu_{\omega}}\left(\bigvee_{i=0}^{n-1}\left(\varphi_{\omega}^{i}\right)^{-1} \xi_{\theta^{i}(\omega)}\right)
$$


for P.a.e. $\omega$ in $\Omega$.

Finally, the entropy of the random dynamical system $\varphi$ is defined as $h_{\mu}^{r}(\varphi)=\sup _{\xi} h_{\mu}^{r}(\varphi, \xi)$ where the supremum is taken over all finite measurable partitions of $\Omega \times X$. We also have the following lemma.

Lemma 2.3. Given any partition $\xi$ of $\Omega \times X$, the mapping $\mu \mapsto h_{\mu}^{r}(\varphi, \xi)$ is affine.

Proof. Let $\mu, \nu \in I_{\mathbb{P}}(\varphi)$ and $\lambda \in[0,1]$. First note that, if $\left\{\mu_{\omega}\right\}_{\omega \in \Omega}$ and $\left\{\nu_{\omega}\right\}_{\omega \in \Omega}$ are disintegrations of $\mu$ and $\nu$ respectively, then $\left\{\lambda \mu_{\omega}+(1-\lambda) \nu_{\omega}\right\}_{\omega \in \Omega}$ is a disintegration of $\lambda \mu+(1-\lambda) \nu$. On the other hand, for $\omega \in \Omega$ and $n \geq 1$, since the function $\eta(s)=-s \log s$ is concave and $\gamma(s)=\log s$ is increasing, we have:

$$
0 \leq H_{\lambda \mu_{\omega}+(1-\lambda) \nu_{\omega}}\left(\xi_{\omega}^{n}\right)-\lambda H_{\mu_{\omega}}\left(\xi_{\omega}^{n}\right)-(1-\lambda) H_{\nu_{\omega}}\left(\xi_{\omega}^{n}\right) \leq \log 2
$$

where $\xi_{\omega}^{n}:=\bigvee_{i=0}^{n-1}\left(\varphi_{\omega}^{i}\right)^{-1} \xi_{\theta^{i}(\omega)}$. Finally, the previous inequalities easily result in

$$
h_{\lambda \mu+(1-\lambda) \nu}^{r}(\varphi, \xi)=\lambda h_{\mu}^{r}(\varphi, \xi)+(1-\lambda) h_{\nu}^{r}(\varphi, \xi)
$$

which completes the proof.

At the end of this section, we review the Abramov-Rokhlin theorem which connects the entropy of a random dynamical system $h_{\mu}^{r}(\varphi)$ to the classical Kolmogrov entropies $h_{\mu}(\Phi)$ and $h_{\mathbb{P}}(\theta)$.

Theorem 2.4. [3] Let $\varphi:(\Omega, \mathcal{F}, \mathbb{P}) \rightarrow C(X, X)$ be a random dynamical system on $X$ over $(\Omega, \mathcal{F}, \mathbb{P}, \theta)$ and $\Phi$ be the corresponding skew product. Then, we have

$$
h_{\mu}(\Phi)=h_{\mu}^{r}(\varphi)+h_{\mathbb{P}}(\theta) .
$$

\section{Local Approach}

In this section, we present a local approach to the entropy of random dynamical systems. It obviously results in a local entropy for classical dynamical systems as a special case.

In the rest of paper, $X$ is a compact metric space, $\varphi:(\Omega, \mathcal{F}, \mathbb{P}) \rightarrow C(X, X)$ is a continuous random dynamical system on $X$ over $(\Omega, \mathcal{F}, \mathbb{P}, \theta)$ and $\Phi$ is the corresponding skew product.

Definition 3.1. For $\omega \in \Omega, x \in X$ and $D \in \mathcal{F} \times \mathcal{B}_{X}$, set

$$
\gamma_{\varphi}(\omega, x, D):=\limsup _{n \rightarrow \infty} \frac{1}{n} \operatorname{card}\left(\left\{0 \leq j \leq n-1: \varphi_{\omega}^{j}(x) \in D_{\theta^{j}}(\omega)\right\}\right) .
$$

Definition 3.2. Let $\omega \in \Omega, x \in X$ and $\xi$ be a finite measurable partition of $\Omega \times X$. Let $g:[0,1] \rightarrow \mathbb{R}$ be the function defined by $g(0)=0$ and $g(s)=-s \log s(s \in(0,1])$. Set

$$
\Gamma_{\varphi}(\omega, x ; \xi):=\sum_{D \in \xi} g\left(\gamma_{\varphi}(\omega, x, D)\right) .
$$

Definition 3.3. The local entropy map of the random dynamical system $\varphi$ over $(\Omega, \mathcal{F}, \mathbb{P}, \theta)$ with respect to the partition $\xi$, is a map $\mathcal{J}_{\varphi}^{r}(\cdot, \cdot ; \xi): \Omega \times X \rightarrow \mathbb{R}$ defined by

$$
\mathcal{J}_{\varphi}^{r}(\omega, x ; \xi):=\mathcal{J}_{\varphi}(\omega, x ; \xi)-h_{\mathbb{P}}(\theta),
$$

where

$$
\mathcal{J}_{\varphi}(\omega, x ; \xi):=\limsup _{n \rightarrow \infty} \frac{1}{n} \Gamma_{\varphi}\left(\omega, x ; \bigvee_{i=0}^{n-1}\left(\Phi^{i}\right)^{-1} \xi\right)
$$


The conditional version of (3.1) is also defined as follows:

$$
\Gamma_{\varphi}(\omega, x ; \xi \mid \eta):=-\sum_{A \in \xi, B \in \eta} \gamma_{\varphi}(\omega, x, A \cap B) \log \frac{\gamma_{\varphi}(\omega, x, A \cap B)}{\gamma_{\varphi}(\omega, x, B)}
$$

The following proposition states some of the properties of the previous quantities.

Proposition 3.4. Let $\omega \in \Omega, x \in X$ and $\xi, \eta$ and $\zeta$ be finite measurable partitions of $\Omega \times X$. Then,

(1) $0 \leq \Gamma_{\varphi}(\omega, x ; \xi \mid \zeta) \leq \Gamma_{\varphi}(\omega, x ; \xi \vee \eta \mid \zeta)$

(2) $\Gamma_{\varphi}(\omega, x ; \xi \vee \eta \mid \zeta) \geq \Gamma_{\varphi}(\omega, x ; \xi \mid \zeta)+\Gamma_{\varphi}(\omega, x ; \eta \mid \xi \vee \zeta)$

(3) If $\xi<\eta$ then $\Gamma_{\varphi}(\omega, x ; \xi \mid \zeta) \leq \Gamma_{\varphi}(\omega, x ; \eta \mid \zeta)$.

\section{Proof.}

(1) First note that, for $\omega \in \Omega$ and $x \in X$, we have:

$$
\sum_{B \in \eta} \gamma_{\varphi}(\omega, x, A \cap B \cap C) \geq \gamma_{\varphi}(\omega, x, A \cap C)
$$

Applying (3.2), we will have:

$$
\begin{aligned}
\Gamma_{\varphi}(\omega, x ; \xi \vee \eta \mid \zeta) & =-\sum_{A \in \xi, B \in \eta, C \in \zeta} \gamma_{\varphi}(\omega, x, A \cap B \cap C) \log \frac{\gamma_{\varphi}(\omega, x, A \cap B \cap C)}{\gamma_{\varphi}(\omega, x, C)} \\
& \geq-\sum_{A \in \xi, B \in \eta, C \in \zeta} \gamma_{\varphi}(\omega, x, A \cap B \cap C) \log \frac{\gamma_{\varphi}(\omega, x, A \cap C)}{\gamma_{\varphi}(\omega, x, C)} \\
& \geq-\sum_{A \in \xi, C \in \zeta} \gamma_{\varphi}(\omega, x, A \cap C) \log \frac{\gamma_{\varphi}(\omega, x, A \cap C)}{\gamma_{\varphi}(\omega, x, C)} \\
& =\Gamma_{\varphi}(\omega, x ; \xi \mid \zeta) .
\end{aligned}
$$

(2) Let $\xi, \eta$ and $\zeta$ be finite measurable partition of $\Omega \times X$. Without loss of generality, we may assume that $\gamma_{\varphi}(\omega, x, B)>0$ for all sets in $\xi, \eta$ and $\zeta$. Now, we have:

$$
\Gamma_{\varphi}(\omega, x ; \xi \vee \eta \mid \xi)=-\sum_{A \in \xi, B \in \eta, C \in \zeta} \gamma_{\varphi}(\omega, x, A \cap B \cap C) \log \frac{\gamma_{\varphi}(\omega, x, A \cap B \cap C)}{\gamma_{\varphi}(\omega, x, C)}
$$

Since

$$
\frac{\gamma_{\varphi}(\omega, x, A \cap B \cap C)}{\gamma_{\varphi}(\omega, x, C)}=\frac{\gamma_{\varphi}(\omega, x, A \cap B \cap C)}{\gamma_{\varphi}(\omega, x, A \cap C)} \cdot \frac{\gamma_{\varphi}(\omega, x, A \cap C)}{\gamma_{\varphi}(\omega, x, C)}
$$


(Note that, if $\gamma_{\varphi}(\omega, x, A \cap C)=0$, the left hand side is zero and we need not consider it), then

$$
\begin{aligned}
\Gamma_{\varphi}(\omega, x ; \xi \vee \eta \mid \zeta) & =-\sum_{A \in \xi, B \in \eta, C \in \zeta} \gamma_{\varphi}(\omega, x, A \cap B \cap C) \log \frac{\gamma_{\varphi}(\omega, x, A \cap C)}{\gamma_{\varphi}(\omega, x, C)} \\
& -\sum_{A \in \xi, B \in \eta, C \in \zeta} \gamma_{\varphi}(\omega, x, A \cap B \cap C) \log \frac{\gamma_{\varphi}(\omega, x, A \cap B \cap C)}{\gamma_{\varphi}(\omega, x, A \cap C)} \\
& \geq-\sum_{A \in \xi, C \in \zeta} \gamma_{\varphi}(\omega, x, A \cap C) \log \frac{\gamma_{\varphi}(\omega, x, A \cap C)}{\gamma_{\varphi}(\omega, x, C)}+\Gamma_{\varphi}(\omega, x ; \eta \mid \xi \vee \zeta) \\
& =\Gamma_{\varphi}(\omega, x ; \xi \mid \zeta)+\Gamma_{\varphi}(\omega, x ; \eta \mid \xi \vee \zeta) .
\end{aligned}
$$

(3) Since $\xi<\eta$ then $\xi \vee \eta=\eta$. Therefore, applying part (ii),

$$
\Gamma_{\varphi}(\omega, x ; \eta \mid \zeta)=\Gamma_{\varphi}(\omega, x ; \xi \vee \eta \mid \zeta) \geq \Gamma_{\varphi}(\omega, x ; \xi \mid \zeta)+\Gamma_{\varphi}(\omega, x ; \eta \mid \xi \vee \zeta) \geq \Gamma_{\varphi}(\omega, x ; \xi \mid \zeta)
$$

In the theory of deterministic dynamical systems, a measure-theoretic dynamical system $(Y, \mathcal{B}, \nu, g)$ is said to be a factor of $(X, \mathcal{A}, \mu, f)$ if there is a measure preserving map $\pi$ : $(X, \mathcal{A}, \mu) \rightarrow(Y, \mathcal{B}, \nu)$ such that the following diagram is commutative $\mu$-almost everywhere:

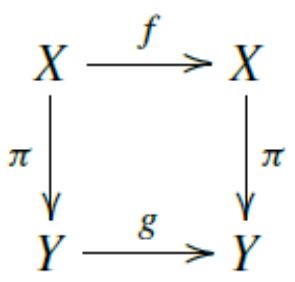

Figure 1

It is proved that, whenever $(X, \mathcal{A}, \mu, f)$ and $(Y, \mathcal{B}, \nu, g)$ are Lebesgue spaces, if $g$ is a factor of $f$ via the measurable map $\pi: X \rightarrow Y$ such that $\operatorname{card}\left(\pi^{-1}\{y\}\right)$ is finite for $\nu$-almost every $y$ in $Y$ then $h_{\mu}(f)=h_{\nu}(g)[15]$.

A similar result is formulated and proved for random dynamical systems in [15]. We formulate and state a local version of the invariance of entropy for factors.

Proposition 3.5. Let $\varphi:(\Omega, \mathcal{F}, \mathbb{P}) \rightarrow C(X, X)$ defined by $\omega \longmapsto \varphi_{\omega}$ and $\psi:(\Omega, \mathcal{F}, \mathbb{P}) \rightarrow$ $C(Y, Y)$ defined by $\omega \longmapsto \psi_{\omega}$ be two random dynamical systems over $(\Omega, \mathcal{F}, \mathbb{P}, \theta)$. Assume that there exists a family of measurable maps $\left\{\pi_{\omega}: X \rightarrow Y\right\}_{\omega \in \Omega}$ such that, for every $\omega \in \Omega$, the following diagram is commutative:

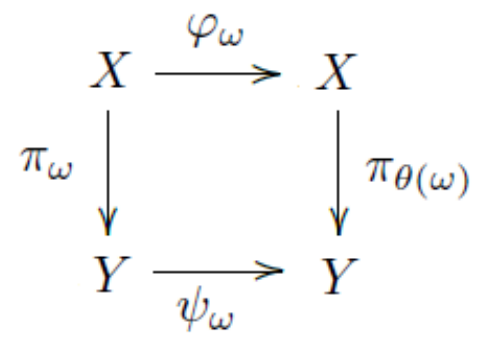

Figure 2 
where $\Pi: \Omega \times X \rightarrow \Omega \times Y,(\omega, x) \longmapsto\left(\omega, \pi_{\omega}(x)\right)$ is a measurable map. Then, for every finite measurable partition $\xi$ of $\Omega \times Y$, we have

$$
\mathcal{J}_{\psi}^{r}(\cdot, \cdot ; \xi) \circ \Pi=\mathcal{J}_{\varphi}^{r}\left(\cdot, \cdot ; \Pi^{-1}(\xi)\right)
$$

or equivalently, the following diagram is commutative:

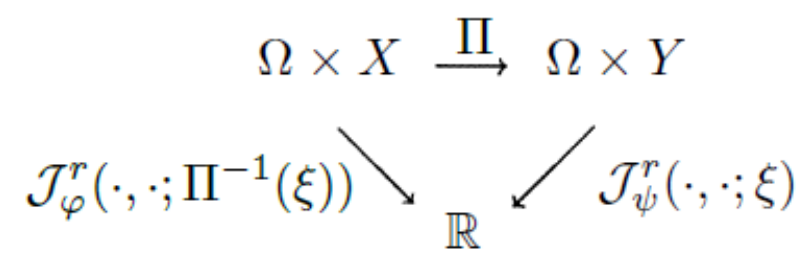

Figure 3

Proof. Let $\Phi$ and $\Psi$ be the corresponding skew products of $\varphi$ and $\psi$ respectively. Since the diagram in Figure 2 is commutative, we have $\Psi \circ \Pi=\Pi \circ \Phi$. Let $D \in \mathcal{F} \times \mathcal{B}_{Y}$ and $(\omega, x) \in \Omega \times X$. We have

$$
\begin{aligned}
\gamma_{\psi}\left(\omega, \pi_{\omega}(x), D\right) & =\limsup _{n \rightarrow+\infty} \frac{1}{n} \operatorname{card}\left(\left\{0 \leq j \leq n-1: \psi_{\omega}^{j}\left(\pi_{\omega}(x)\right) \in D_{\theta^{j}(\omega)}\right\}\right) \\
& =\limsup _{n \rightarrow+\infty} \frac{1}{n} \sum_{j=0}^{n-1} \chi_{D}\left(\Psi^{j}\left(\omega, \pi_{\omega}(x)\right)\right) \\
& =\limsup _{n \rightarrow+\infty} \frac{1}{n} \sum_{j=0}^{n-1} \chi_{D}\left(\left(\Psi^{j} \circ \Pi\right)(\omega, x)\right) \\
& =\limsup _{n \rightarrow+\infty} \frac{1}{n} \sum_{j=0}^{n-1} \chi_{D}\left(\left(\Pi \circ \Phi^{j}\right)(\omega, x)\right) \\
& =\limsup _{n \rightarrow+\infty} \frac{1}{n} \sum_{j=0}^{n-1} \chi_{\Pi^{-1}(D)}\left(\left(\Phi^{j}\right)(\omega, x)\right) \\
& =\gamma_{\varphi}\left(\omega, x, \Pi^{-1}(D)\right) .
\end{aligned}
$$

Therefore, given any measurable partition $\xi$ of $\Omega \times Y$, we conclude that

$$
\Gamma_{\psi}\left(\omega, \pi_{\omega}(x) ; \xi\right)=\Gamma_{\varphi}\left(\omega, x ; \Pi^{-1}(\xi)\right)
$$


Replacing $\xi$ by $\bigvee_{j=0}^{n-1} \Psi^{-j}(\xi)$ in (3.3), we will have

$$
\begin{aligned}
\mathcal{J}_{\psi}\left(\omega, \pi_{\omega}(x) ; \xi\right) & =\limsup _{n \rightarrow+\infty} \frac{1}{n} \Gamma_{\psi}\left(\omega, \pi_{\omega}(x) ; \bigvee_{j=0}^{n-1} \Psi^{-j}(\xi)\right) \\
& =\limsup _{n \rightarrow+\infty} \frac{1}{n} \Gamma_{\varphi}\left(\omega, x ; \Pi^{-1}\left(\bigvee_{j=0}^{n-1} \Psi^{-j}(\xi)\right)\right. \\
& =\limsup _{n \rightarrow+\infty} \frac{1}{n} \Gamma_{\varphi}\left(\omega, x ; \bigvee_{j=0}^{n-1} \Pi^{-1}\left(\Psi^{-j}(\xi)\right)\right. \\
& =\limsup _{n \rightarrow+\infty} \frac{1}{n} \Gamma_{\varphi}\left(\omega, x ; \bigvee_{j=0}^{n-1} \Phi^{-j}\left(\Pi^{-1}(\xi)\right)\right. \\
& =\mathcal{J}_{\varphi}\left(\omega, x ; \Pi^{-1}(\xi)\right) .
\end{aligned}
$$

This completes the proof.

Now, we are ready to show that the map $\mathcal{J}_{\varphi}^{r}(\cdot, \cdot ; \xi)$ is indeed a local entropy map.

Theorem 3.6. For any $\mu \in I_{\mathbb{P}}(\varphi)$ we have:

$$
h_{\mu}^{r}(\varphi)=\sup _{\xi} \int_{\Omega \times X} \mathcal{J}_{\varphi}^{r}(\omega, x ; \xi) d \mu(\omega, x)
$$

where the supremum is taken over all finite measurable partitions of $\Omega \times X$.

Proof. First, let $\mu \in E_{\mathbb{P}}(\varphi)$. Let $A \in \mathcal{F} \times \mathcal{B}_{X}$, then by Birkhoff ergodic theorem,

$$
\begin{aligned}
\gamma_{\varphi}(\omega, x, A) & =\limsup _{n \rightarrow \infty} \frac{1}{n} \operatorname{card}\left(\left\{0 \leq j \leq n-1: \varphi_{\omega}^{j}(x) \in A_{\theta^{j}(\omega)}\right\}\right) \\
& =\limsup _{n \rightarrow \infty} \frac{1}{n} \sum_{j=0}^{n-1} \chi_{A_{\theta^{j}(\omega)}}\left(\varphi_{\omega}^{j}(x)\right) \\
& =\limsup _{n \rightarrow \infty} \frac{1}{n} \sum_{j=0}^{n-1} \chi_{A}\left(\theta^{j}(\omega), \varphi^{j}(x)\right) \\
& =\limsup _{n \rightarrow \infty} \frac{1}{n} \sum_{j=0}^{n-1} \chi_{A}\left(\Phi^{j}(\omega, x)\right) \\
& =\int_{\Omega \times X} \chi_{A} d \mu=\mu(A)
\end{aligned}
$$

for $\mathbb{P}$.a.e. $\omega \in \Omega$.

For any finite measurable partition $\xi$ of $\Omega \times X,(3.4)$ results in

$$
\Gamma_{\varphi}(\omega, x ; \xi)=H_{\mu}(\xi)
$$

for P.a.e. $\omega \in \Omega$. 
For $n \geq 1$, replacing $\xi$ by $\bigvee_{i=0}^{n-1}\left(\Phi^{i}\right)^{-1} \xi$ in (3.5), there exist a $\mathbb{P}$-measurable set $\Lambda_{n} \subset \Omega \times X$ with $\mu\left(\Lambda_{n}\right)=1$ and

$$
\frac{1}{n} \Gamma_{\varphi}\left(\omega, x ; \bigvee_{i=0}^{n-1}\left(\Phi^{i}\right)^{-1} \xi\right)=\frac{1}{n} H_{\mu}\left(\bigvee_{i=0}^{n-1}\left(\Phi^{i}\right)^{-1} \xi\right)
$$

for $(\omega, x) \in \Lambda_{n}$.

Set $\Lambda:=\bigcap_{n=1}^{\infty} \Lambda_{n}$. Then $\mu(\Lambda)=1$ and (3.6) holds for any $(\omega, x) \in \Lambda$. Letting $n \rightarrow \infty$ in (3.6), we conclude that

$$
\mathcal{J}_{\varphi}(\omega, x ; \xi)=h_{\mu}(\varphi, \xi)
$$

for any $(\omega, x) \in \Lambda$. Therefore,

$$
\mathcal{J}_{\varphi}^{r}(\omega, x ; \xi)=h_{\mu}(\Phi, \xi)-h_{\mathbb{P}}(\theta)
$$

for any $(\omega, x) \in \Lambda$.

Integrating both sides of (3.7), we will have:

$$
\int_{\Omega \times X} \mathcal{J}_{\varphi}^{r}(\omega, x ; \xi) d \mu(\omega, x)=\int_{\Lambda} \mathcal{J}_{\varphi}^{r}(\omega, x ; \xi) d \mu(\omega, x)=h_{\mu}(\Phi, \xi)-h_{\mathbb{P}}(\theta) .
$$

Now, let in general, $\mu \in I_{\mathbb{P}}(\varphi)$. Let $\mu=\int_{E_{\mathbb{P}}(\varphi)} \nu d \tau(\nu)$ be the ergodic decomposition of $\mu$. Applying (3.8) and Jacob's theorem, we will have:

$$
\begin{aligned}
\int_{\Omega \times X} \mathcal{J}_{\varphi}^{r}(\omega, x ; \xi) d \mu(\nu, x) & =\int_{\Omega \times X} \mathcal{J}_{\varphi}(\omega, x ; \xi) d \mu(\omega, x)-h_{\mathbb{P}}(\theta) \\
& =\int_{E_{\mathbb{P}}(\varphi)}\left(\int_{\Omega \times X} \mathcal{J}_{\varphi}(\omega, x ; \xi) d \nu(\omega, x)\right) d \tau(\nu)-h_{\mathbb{P}}(\theta) \\
& =\int_{E_{\mathbb{P}}(\varphi)} h_{\nu}(\Phi, \xi) d \tau(\nu)-h_{\mathbb{P}}(\theta) \\
& =h_{\mu}(\Phi, \xi)-h_{\mathbb{P}}(\theta) .
\end{aligned}
$$

Finally, the result follows by taking supremume over all measurable partitions $\xi$ of $\Omega \times X$ and the Abramov-Rokhlin theorem.

\section{DisCUSSION AND CONCLUDING REMARKS}

Local entropies are applied in multifractal analysis to characterize dynamical systems [4, $22,23,33]$. It studies the dimensional properties of the level sets of certain functions like local entropies, using either Hausdorff dimension or topological entropy in the sense of Bowen $[9,21]$.

Local entropies may also be applied to measure the amount of information generated by a system in a certain area of the space rather that the whole space. As an example, one may see the definition of information content of a molecular structure via local entropies in [24].

As in the entropy theory of classical dynamical systems, we presented a local view to the concept of entropy of random dynamical systems. We introduced a map $\mathcal{J}_{\varphi}^{r}(\cdot, \cdot ; \xi): \Omega \times X \rightarrow \mathbb{R}$ which plays the role of a local entropy for the random dynamical system $\varphi$ over $(\Omega, \mathcal{F}, \mathbb{P}, \theta)$, in the sense that, the entropy of the random dynamical system $\varphi$ may be extracted by integrating the introduced map $\mathcal{J}_{\varphi}^{r}(\cdot, \cdot ; \xi)$. 


\section{Acknowledgments}

The authors would like to thank the referees for their comprehensive and useful comments which helped the improvement of this work to the present form.

\section{REFERENCES}

[1] L. Arnold, Random Dynamical Systems, Springer Monographs in Mathematics (Corrected 2nd Printing, 2003).

[2] L. Arnold, B. Schmalfuss, Fixed Points and Attractors for Random Dynamical Systems, IUTAM Symposium on Advances in Nonlinear Stochastic Mechanics. Solid Mechanics and its Applications, bf 47. Springer, Dordrecht.

[3] T. Bogenschutz, H. Crauel, The Abramov-Rokhlin formula, In: Krengel U., Richter K., Warstat V. (eds) Ergodic Theory and Related Topics III. Lecture Notes in Mathematics, vol 1514. Springer, Berlin, Heidelberg. https://doi.org/10.1007/BFb0097526.

[4] L. Barreira, Ya. Pesin, J. Schemling, On a general concept of multifractality: Multifractal spectra for dimensions, entropies, and Lyapunov exponents. Multifractal rigidity, Chaos 7 (1), 27-38, 1997.

[5] L. Breiman, The individual theorem of information theory. Ann. of Math. Stat. 28 (1957), 809-811, errata 31, 809-810, 1960.

[6] M. Brin, A. Katok, On local entropy in geometric dynamics, 30-38. New York, Springer-Verlag, (Lecture Notes in Mathematics 1007), 1983.

[7] T. Bogenschutz, Entropy, pressure and a variational principle for random dynamical systems, Random Comput. Dynam., 1, 199-116, 1992.

[8] T. Bogenschutz, Equilibrium states for random dynamical systems, Ph.D. Thesis, Bremen University, 1993.

[9] R. Bowen, Topological entropy for non-compact sets, Trans. Amer. Math. Soc., 184, 125-136, 1973.

[10] A. Katok, Entropy and closed geodesics, Erg. Th. Dyn. Sys. 2, 339-365, 1983.

[11] Y. Kifer, Ergodic Theory of Random Transformations, Springer, Progress in Probability and Statistics, 10, 13-25, 1986.

[12] Y. Kifer, P. D. Liu, Random Dynamical Systems, Handbook of Dynamical Systems, vol. 1B, eds, B. Hasselblatt and A. Katok, Elsevier, 379-499, 2006.

[13] A. N. Kolmogorov, New metric invariant of transitive dynamical systems and endomorphisms of Lebesgue spaces, Doklady of Russian Academy of Sciences, 119, N5, 861-864, 1958.

[14] P. D. Liu, Dynamics of random transformations: smooth ergodic theory, Ergo. Theo. Dyn. Syst., 21, 1279-1319, 2001.

[15] P. D. Liu, A note on the entropy of factors of random dynamical systems, Ergodic Theory and Dynamical Systems, 25, 593-603 doi:10.1017/S0143385704000586.

[16] R. Mañé, Ergodic theory and differentiable dynamics. Springer-Verlag, Berlin, Heidelberg, New York, 1987.

[17] R. Mañé, On the topological entropy of geodesic flows. J. Differential Geometry, 45, 74-93, 1997.

[18] B. McMillan, The basic theorems of information theory. Ann. of Math. Statistics 24, 196-219, 1953.

[19] R. Phelps, Lectures on Choquet's Theorem, Van Nostrand, Princeton, N.J., 1966.

[20] Ya. Pesin, Characteristic Lyapunov exponents and smooth ergodic theory. Russian Math. Surveys 32, 54-114, 1977.

[21] Ya. Pesin, Dimension Theory in Dynamical Systems: Contemporary Views and Applications, Chicago Lectures in Mathematics, University of Chicago Press, Chicago, IL, 1997.

[22] Ya. Pesin, H. Weiss, A multifractal analysis of equilibrium measures for conformal expanding maps and Moran-like geometric constructions, J. Stat. Phys. 86 (1-2), 233-275, 1997.

[23] Ya. Pesin, H. Weiss, The multifractal analysis of Gibbs measures: Motivation, mathematical foundation, and examples, Chaos 7 (1), 89-106, 1997.

[24] M. Rahimi, P. Nasertayoob, Dynamical Information Content of the Molecular Structures: A Quantum Theory of Atoms in Molecules (QTAIM) Approach, MATCH Commun. Math. Comput. Chem. 67 109-126, 2012.

[25] M. Rahimi, A local approach to g-entropy, Kybernetika, 51, 231-245, 2015.

[26] M. Rahimi, M. Mohammadi Anjedani, A local view on the Hudetz correction of the Yager entropy of dynamical systems, International Journal of General Systems, DOI: 10.1080/03081079.2018.1552688, 2018 . 
[27] M. Rahimi, A. Shakouri, On Hudetz entropy localization, Fuzzy Sets and Systems, https:DOI: 10.1016/j.fss.2018.11.005, 2019.

[28] V . Rokhlin, On the entropy of a metric automorphism, Dokl . Akad . Nauk. SSSR, 124,980-983, 1959.

[29] D. Ruelle, An inequality for the entropy of differential maps. Bol. Soc. Bras. de Mat. 9, 83-87, 1978.

[30] C. Shannon, A mathematical theory of communication. Bell Syst. Tech. Journal 27, 379-423, 623-656, 1948.

[31] M. Scheutzow, Attractors for Ergodic and Monotone Random Dynamical Systems, Progress in Probability, 59, 331-344, 2007.

[32] Ya. G. Sinai, On the notion of entropy of a dynamical system, Doklady of Russian Academy of Sciences, 124, 768-771, 1959.

[33] F. Takens, E. Verbitski, Multifractal Analysis of Local Entropies for Expansive Homeomorphisms with Specification, Commun. Math. Phys. 203, 593 - 612, 1999.

[34] Y. J. Zhu, On local entropy of random transformations, Stoch. Dyn., 8, 197-207, 2008.

[35] Y. J. Zhu, Two Notes on Measure-Theoretic Entropy of Random Dynamical Systems, Acta Mathematica Sinica, English Series, 25, No. 6, 961-970, 2009.

(Mehdi Rahimi) Department of Mathematics, Faculty of Science, University of Qom, Qom, IRAN.

Email address: m10.rahimi@gmail.com

(Ahmad Shakouri) Department of Mathematics, Faculty of Science, University of Qom, Qom, IRAN.

Email address: shakourisamad@gmail.com

(Mohammad Mohammadi Anjedani) Department of Mathematics, Faculty of Science, University OF QOM, QOM, IRAN.

Email address: mm_math67@yahoo.com 